\title{
SHELL DYNAMICS WITH THREE-DIMENSIONAL DEGENERATE FINITE ELEMENTS
}

\author{
T. KANT, $\$$ S. Kumar and U. P. Singh \\ Department of Civil Engineering, Indian Institute of Technology, Powai, Bombay-400 076, India
}

(Received 17 January 1992)

\begin{abstract}
An explicitly through the thickness integrated two-dimensional version of the three-dimensional degenerated shell element is formulated here to study the dynamics of elastic shells. A nine-noded quadrilateral Lagrangian element is used with five degrees of freedom per node. A specialized mass diagonalization scheme, developed by Hinton, Rock and Zienkiewicz, is used which conserves the total mass of the element and also includes the effects of the rotary inertia terms. Hamilton's principle is used to derive the equations of motion. Mode superposition coupled with Duhamel's integral is first employed to obtain a solution of the equations of motion in time. Mode shapes and frequencies are computed by subspace iteration technique. Direct time integration using the implicit Newmark- $\beta$ method is also carried out. Several examples are presented and the results obtained by mode superposition and direct time integration methods are compared.
\end{abstract}

\section{INTRODUCTION}

The study of dynamic behaviour of shell structures, the use of which in engineering and other fields has been increasing at a remarkable rate since the start of this century, is of crucial importance. This is so because often such structures are subjected to timevarying loadings such as impact, explosion or seismic effects.

This paper places emphasis on elasto-dynamic as well as seismic analyses of shell structures using two-dimensional (2D) degenerated shell finite elements. In the degeneration procedure, the threedimensional (3D) theory is reduced or degenerated to a shell theory simultaneously with the finite element discretization. The finite element analysis of shells took a new direction after the development of the degenerated shell element by Ahmad et al. [1]. After this a great deal of research activity in the last 20 years has led to improved versions of the original degenerated shell element. The Gaussian quadrature rule for the evaluation of the energy terms in the eight-noded quadratic serendipity quadrilateral element of Ahmad et al. was $3 \times 3$ for all the types, namely membrane, flexure and transverse shear energies. This was the minimum order of integration required to produce exact results and was therefore considered appropriate. The results however, were found to be reasonable only for thick shells. In the case of moderately thick and thin shells, numerical results departed considerably from closed-form analytical solutions. In fact the element was found to be too stiff with a very slow rate of convergence in such cases.

† To whom all correspondence should be addressed.
Zienkiewicz et al. [2] introduced the so-called uniform reduced integration technique in which a Gaussian quadrature order of $2 \times 2$ was used. The results, without doubt, improved for moderate to thin shells, but below a certain thinness the element behaviour was erratic. This erratic behaviour led to a near abandonment of the eight-noded serendipity element. Later a heuristic explanation was provided by Malkus and Hughes [3]. The stiff behaviour of the structure was attributed to the now popularly called 'shear and membrane locking' behaviour. This locking behaviour occurred because of the inability of the element to model deformed states in which membrane strains and transverse shear strains vanished.

Hughes et al. [4] then introduced the selective reduced integration technique in which a reduced order of integration is used for membrane and shear energy terms to underestimate their effects in thin situations. The performance of the nine-node quadrilateral Lagrangian element has been found to be most satisfactory for both thick and thin shell problems in recent years. A good description of the developments in chronological order can be found in Pugh et al. [5]. Parisch [6], Belytschko et al. [7], Milford and Schnobrich [8] and Kant and Datye [9]. Recently, substitute shear strain fields have been used by Bathe and Dvorkin [10] to overcome shear locking. Using a similar approach Huang and Hinton [11] have developed eight- and nine-noded Mindlin plate elements and degenerated shell elements with substitute shear-strain fields.

\section{THEORETICAL FORMULATION}

The theory on which the finite element formulation is based is given here for the sake of completeness; 
however, a general presentation is available elsewhere [9].

\subsection{Coordinate systems and element geometry}

Four different sets of coordinate systems are employed as shown in Fig. 1. The global coordinate system $(X, Y, Z)$ is used to define structure geometry. The shape functions are expressed in natural curvilinear coordinates $(\xi, \eta)$. A set of tangent vectors to mid-surface $\overline{\mathbf{V}}_{\xi}, \overline{\mathbf{V}}_{\eta}$ is used to get nodal and local direction cosines where

$$
\begin{aligned}
\overline{\mathbf{V}}_{\xi} & =\frac{\partial \mathbf{X}}{\partial \xi} \mathbf{i}+\frac{\partial \mathbf{Y}}{\partial \xi} \mathbf{j}+\frac{\partial \mathbf{Z}}{\partial \xi} \mathbf{k} \\
& =\left(\sum \frac{\partial \mathbf{N}_{i}}{\partial \xi} \mathbf{X}_{i}\right) \mathbf{i}+\left(\sum \frac{\partial \mathbf{N}_{i}}{\partial \xi} \mathbf{Y}_{i}\right) \mathbf{j}+\left(\sum \frac{\partial \mathbf{N}_{i}}{\partial \xi} \mathbf{Z}_{i}\right) \mathbf{k} \\
\overline{\mathbf{V}}_{\eta} & =\frac{\partial \mathbf{X}}{\partial \eta} \mathbf{i}+\frac{\partial \mathbf{Y}}{\partial \eta} \mathbf{j}+\frac{\partial \mathbf{Z}}{\partial \eta} \mathbf{k} \\
& =\left(\sum \frac{\partial \mathbf{N}_{i}}{\partial \eta} \mathbf{X}_{i}\right) \mathbf{i}+\left(\sum \frac{\partial \mathbf{N}_{i}}{\partial \eta} \mathbf{Y}_{i}\right) \mathbf{j}+\left(\sum \frac{\partial \mathbf{N}_{i}}{\partial \eta} \mathbf{Z}_{i}\right) \mathbf{k} .
\end{aligned}
$$

The nodal coordinate set $\left(x_{n}, y_{n}, z_{n}\right)$ with $\mathbf{i}_{n}, \mathbf{j}_{n}, \mathbf{k}_{n}$ as unit vectors is defined at each nodal point. The vector $\mathbf{k}_{n}$ is oriented along $\mathbf{V}_{\perp}$ [given by $\left(\overline{\mathbf{V}}_{\xi} \times \bar{\nabla}_{\eta}\right)$ ] and $\mathbf{i}_{n}$ can be either along $\nabla_{\xi}$ or along a vector given by $\left(\mathbf{j} \times \mathbf{k}_{n}\right)$. The vector $\mathbf{j}_{n}$ is given by $\left(\mathbf{k}_{n} \times \mathbf{i}_{n}\right)$. The local coordinate set $\left(x_{x}, y_{\gamma}, z_{\gamma}\right)$ is defined at each point on the mid-surface with $\mathbf{i}_{x}, \mathbf{j}_{x}, \mathbf{k}_{x}$ as unit vectors. The vector $\mathbf{k}_{x}$ is oriented along $\bar{V}_{\perp}, \mathbf{i}_{\alpha}$ along $\overline{\mathbf{V}}_{\xi}$, and $\mathbf{j}_{x}$ is given by $\left(\mathbf{k}_{x} \times \mathbf{i}_{x}\right)$. The coordinates of top $\left(X_{t}, Y_{t}, Z_{t}\right)$ and bottom $\left(X_{h}, Y_{b}, Z_{b}\right)$ surface points at a node are usually necessary to define element geometry. Alternatively, the mid-surface coordinates and corresponding thicknesses can be given. The thickness at node $i$ is

\subsection{Displacement model}

The following displacement model is used

$$
\begin{aligned}
& u_{x}\left(x_{x}, y_{x}, z_{x}, t\right)=u_{o x}\left(x_{x}, y_{x}, t\right)+z_{x} \theta_{y x}\left(x_{x}, y_{x}, t\right) \\
& v_{x}\left(x_{x}, y_{x}, z_{x}, t\right)=v_{n x}\left(x_{x}, y_{x}, t\right)-z_{x} \theta_{x x}\left(x_{x}, y_{x}, t\right) \\
& w_{x}\left(x_{x}, y_{x}, z_{x}, t\right)=w_{o x}\left(x_{x}, y_{x}, t\right)
\end{aligned}
$$

in which $t$ is time, $u_{o x}, v_{o x}$ and $w_{o x}$ are the in-plane and transverse displacements of a point $\left(x_{x}, y_{x}\right)$ on the mid-surface respectively, and $\theta_{x x}$ and $\theta_{y x}$ are the vector rotations of normal to the mid-surface about the $x_{x}$ and $y_{x}$ axes, respectively. The parameters $u_{x}, v_{x}$ and $w_{x}$ are the displacement components in the $x_{x}, y_{x}$ and $z_{z}$ directions, respectively of a generic point.

\subsection{Strain expressions}

In order to easily deal with the thin shell assumption of zero normal stress in the $z$ direction $\left(\sigma_{z x}=0\right)$, the strain components should be defined in terms of a local coordinate set of axes $x_{x}, y_{x}, z_{x}$. In the present model there are eight significant strains over the mid-surface. From eqns (6) we get

$$
\begin{gathered}
\epsilon_{x x}=\epsilon_{x y x}+z \cdot k_{x x} \\
\epsilon_{y x}=\epsilon_{y r x}+z \cdot k_{y x} \\
\epsilon_{z x}=\epsilon_{z x x}=0 \\
\gamma_{x y x}=\epsilon_{x y o x}+z k_{x y} \\
\gamma_{y z x}=\phi_{x} \\
\gamma_{z x x}=\phi_{y}
\end{gathered}
$$

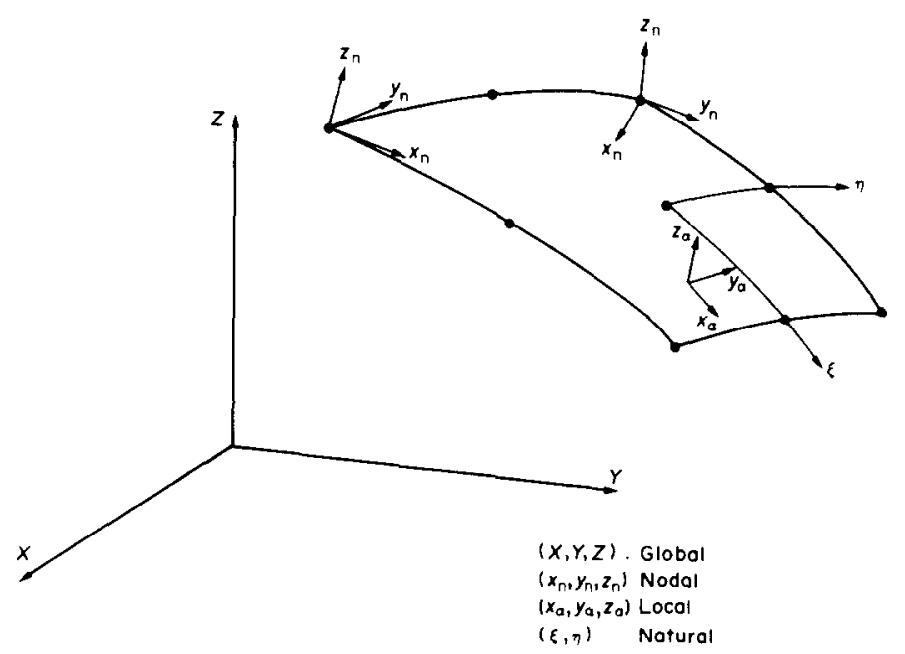

Fig. 1. Coordinate systems. 
in which the mid-surface strains are defined as follows:

$$
\begin{gathered}
\epsilon_{x o x}=\frac{\partial u_{o x}}{\partial x_{x}} \\
\epsilon_{y o x}=\frac{\partial v_{n x}}{\partial y_{x}} \\
\epsilon_{x y a x}=\frac{\partial u_{o x}}{\partial y_{x}}+\frac{\partial v_{o x}}{\partial x_{x}} \\
k_{x x}=\frac{\partial \theta_{y x}}{\partial x_{x}} \\
k_{y x}=-\frac{\partial \theta_{x x}}{\partial y_{x}} \\
k_{x y x}=\frac{\partial \theta_{y x}}{\partial y}-\frac{\partial \theta_{x x}}{\partial x} \\
\phi_{x}=\frac{\partial w_{o x}}{\partial y_{x}}-\theta_{x x} \\
\phi_{y}=\frac{\partial w_{o x}}{\partial x_{x}}+\theta_{y x} .
\end{gathered}
$$

Thus the generalized strain vector and displacement vector corresponding to mid-surface become

$$
\begin{gathered}
\bar{\epsilon}_{x}=\left[\epsilon_{x o x}, \epsilon_{y o x}, \epsilon_{x y o x}, k_{x x}, k_{y x}, k_{x y x}, \phi_{x}, \phi_{y}\right]^{\prime} \\
\mathbf{d}_{x}=\left(u_{o x}, v_{o x}, w_{o x}, \theta_{x x}, \theta_{y z}\right)^{t} .
\end{gathered}
$$

\subsection{Stress-strain relationship}

In confirmity with usual shell assumptions, the normal stress can be assumed small enough to be neglected and the corresponding strain $\epsilon_{z x}$ is equal to zero. The generalized Hooke's law for an isotropic matcrial can be written as

$$
\sigma_{x i}=\mathbf{C}_{i j} \epsilon_{x j} \quad i, j=1,2,3,4,5,
$$

in which the coefficients $\mathbf{C}_{i j}$ constitute the isotropic material stiffness matrix and is given in Appendix A. Also

$$
\begin{aligned}
& \sigma_{x}=\left(\sigma_{x x}, \sigma_{y x}, \tau_{x y x}, \tau_{y=x}, \tau_{x x x}\right)^{\prime} \\
& \epsilon_{x}=\left(\epsilon_{x x}, \epsilon_{y x}, \epsilon_{x y x}, \gamma_{y z x}, \gamma_{z x x}\right)^{\prime} .
\end{aligned}
$$

Stress resultants at the middle surface can be derived using the potential energy expression as follows:

$$
\begin{aligned}
U= & \frac{1}{2} \int_{r} \epsilon_{x}^{\prime} \sigma_{x} \mathrm{~d} v \\
= & \frac{1}{2} \int_{r}\left(\epsilon_{x x} \sigma_{x x}+\epsilon_{y x} \sigma_{y x}+\gamma_{x y x} \tau_{x y x}\right. \\
& \left.+\gamma_{y z x} \tau_{y=x}+\gamma_{z x x} \tau_{z x x}\right) \mathrm{d} v
\end{aligned}
$$

$$
\begin{aligned}
& U=\frac{1}{2} \int_{r}\left(\sigma_{x z}\left(\epsilon_{x o x}+z_{x} k_{x z}\right)+\sigma_{y y x}\left(\epsilon_{y o z}+z_{x} k_{y z}\right)\right. \\
& +\left(\tau_{x y x}\left(\epsilon_{x y o x}+z_{x} k_{x y x}\right)+\tau_{y=x} \phi_{x}\right. \\
& \left.\left.+\tau_{z \mathrm{xx}} \phi_{y}\right) \mathrm{~d} A \cdot \mathrm{d} z\right) \\
& =\frac{1}{2} \int_{A}\left[\epsilon_{x o x} \int_{z_{x}} \sigma_{x x} \mathrm{~d} z+\epsilon_{y o x} \int_{z_{x}} \sigma_{y x} \mathrm{~d} z\right. \\
& +\epsilon_{x y o x} \int_{-\mathrm{x}} \tau_{x y z} \mathrm{~d} z \\
& +k_{x x} \int_{z_{x}} \sigma_{x x} z_{x} \mathrm{~d} z+k_{y z} \int_{z_{x}} \sigma_{y x} z_{x} \mathrm{~d} z \\
& +k_{x y x} \int_{z_{x}} \tau_{x y x} z_{x} \mathrm{~d} z+\phi_{x} \int \tau_{y z x} \mathrm{~d} z \\
& \left.+\phi_{y} \int \tau_{z: x z} \mathrm{~d} z\right] \\
& =\frac{1}{2} \int\left[\epsilon_{x \theta x} \mathbf{N}_{x x}+\epsilon_{y \theta x} \mathbf{N}_{y x}+\epsilon_{x y o x} \mathbf{N}_{x y x}\right. \\
& +k_{x x} \mathbf{M}_{x x}+k_{y x} \mathbf{M}_{y x}+k_{x y z} \mathbf{M}_{x y x} \\
& \left.+\phi_{x x} \mathbf{Q}_{x x}+\phi_{y x} \mathbf{Q}_{y x}\right] \mathrm{d} A .
\end{aligned}
$$

Here $\mathbf{N}_{x z}, \mathbf{N}_{y y}, \mathbf{N}_{x y z}, \mathbf{M}_{x z}, \mathbf{M}_{y z}, \mathbf{M}_{x y z}, \mathbf{Q}_{x x}, \mathbf{Q}_{y x}$ are the stress resultants at the mid-surface. $\mathbf{N}_{x x}, \mathbf{N}_{r x}$ are extensional forces, $\mathbf{Q}_{x z}, \mathbf{Q}_{y z}$ are transverse shear forces, $\mathbf{M}_{x x}, \mathbf{M}_{x x}$ are bending moments, $\mathbf{N}_{x y x}, \mathbf{M}_{x y x}$ are the in-plane shear forces and twisting moments, respectively. The expressions for these forces and moments are given below

$$
\begin{aligned}
\mathbf{N}_{x x} & =\int_{-t_{x} / 2}^{+t_{x} / 2} \sigma_{x x} \mathrm{~d} z \\
\mathbf{N}_{y x} & =\int_{-t_{x} / 2}^{+t_{x} / 2} \sigma_{y x} \mathrm{~d} z \\
\mathbf{N}_{x y x} & =\int_{-t_{x} / 2}^{+t_{x} / 2} \tau_{x y x} \mathrm{~d} z \\
\mathbf{M}_{x x} & =\int_{-t_{x} / 2}^{+t_{x} / 2} \sigma_{x x} z_{x} \mathrm{~d} z \\
\mathbf{M}_{y x} & =\int_{-t_{x} / 2}^{+t_{x} / 2} \sigma_{y x} z_{x} \mathrm{~d} z \\
\mathbf{M}_{x y x} & =\int_{-t_{x} / 2}^{+t_{x} / 2} \tau_{x y x} z_{x} \mathrm{~d} z \\
\mathbf{Q}_{x x} & =\int_{-t_{x} / 2}^{+t_{x} / 2} \tau_{y z x} \mathrm{~d} z \\
\mathbf{Q}_{y x x} & =\int_{-t_{y} / 2}^{+t_{x} / 2} \tau_{x z y} \mathrm{~d} z .
\end{aligned}
$$


After carrying out explicit integration in the thickness direction

$$
\begin{aligned}
& \mathbf{N}_{x}=\overline{\mathbf{D}}_{m x} \bar{\epsilon}_{x} \\
& \mathbf{M}_{x}=\overline{\mathbf{D}}_{h x} \bar{\epsilon}_{x} \\
& \mathbf{Q}_{x}=\overline{\mathbf{D}}_{s x} \bar{\epsilon}_{x},
\end{aligned}
$$

where $\overline{\mathbf{D}}_{m x}, \overline{\mathbf{D}}_{b x}$ and $\overline{\mathbf{D}}_{s \alpha}$ are the membrane, flexural and shear rigidity matrices, respectively, and are given in Appendix B. Also

$$
\bar{\sigma}_{\alpha}=\left[\begin{array}{c}
\mathbf{N}_{\alpha} \\
\mathbf{M}_{\alpha} \\
\mathbf{Q}_{\alpha}
\end{array}\right]=\left[\begin{array}{ccc}
\overline{\mathbf{D}}_{m x} & 0 & 0 \\
0 & \overline{\mathbf{D}}_{b x} & 0 \\
0 & 0 & \overline{\mathbf{D}}_{s \alpha}
\end{array}\right] \cdot \overline{\boldsymbol{\epsilon}}_{\alpha}=\mathbf{D}_{x} \cdot \overline{\boldsymbol{\epsilon}}_{\alpha} .
$$

We can also define

$$
\begin{aligned}
& \bar{\epsilon}_{m x}=\mathbf{L}_{m x} \mathbf{d}_{x} \\
& \bar{\epsilon}_{h x}=\mathbf{L}_{b x} \mathbf{d}_{x} \\
& \bar{\epsilon}_{s x}=\mathbf{L}_{s x} \mathbf{d}_{x} .
\end{aligned}
$$

Here $\mathbf{L}_{m x}, \mathbf{L}_{b x}, \mathbf{L}_{s x}$ are the strain operator matrices in the membranc, bending and shear, respectively, and are listed in Appendix C.

\subsection{Equation of motion}

The mathematical statement of Hamilton's principle is written as

$$
\int_{t_{1}}^{t_{2}} \delta(I-E) \mathrm{d} t=0
$$

where $I$ and $E$ are total potential energy and kinetic energy, respectively. The total potential energy $\Pi$ can be written as

$$
\Pi=U-W
$$

or

$$
\Pi=\frac{1}{2} \int_{x} \epsilon_{x}^{t} \sigma_{z} \mathrm{~d} v-\int_{A} \mathbf{u}_{x}^{t} \mathbf{P}_{x} \mathrm{~d} \boldsymbol{A}
$$

$U$ is the strain energy stored in the shell, $W$ represents work done by externally applied forces. $\mathbf{P}_{\boldsymbol{\alpha}}$ is the vector of force intensities in the direction $\left(x_{x} y_{x} z_{x}\right)$. $\mathbf{u}_{z}$ is the displacement of any generic point in the shell space. After carrying out explicit integration in the through the thickness direction and substituting the expressions for strains and stresses we get

$$
\Pi=\frac{1}{2} \int_{A} \bar{\epsilon}_{x}^{\prime} \bar{\sigma}_{x} \mathrm{~d} A-\int_{A} \mathbf{d}_{x}^{\prime} \mathbf{F} \mathrm{d} A .
$$

F is the vector of the load per unit area corresponding to the direction of generalized displacement vector $\mathbf{d}_{x}$. The kinetic energy $E$ can be written as

$$
E=\frac{1}{2} \int_{r} \dot{\mathbf{u}}^{\prime} \rho \dot{\mathbf{u}} \mathrm{d} v .
$$

$\dot{\mathbf{u}}$ is the velocity vector of any generic point in space and $\rho$ is mass density of the material. An expression for $\mathbf{u}$ can be obtained from eqn (6), and after explicit integration through the thickness we get

$$
E=\frac{1}{2} \int_{A} \dot{\mathbf{d}}_{x}^{\prime} \mathbf{m} \dot{\mathbf{d}}_{x} \mathrm{~d} A
$$

Here $\mathbf{m}$ is the inertia matrix and is of the form

$$
\mathbf{m}=\left[\begin{array}{ccccc}
I_{1} & 0 & 0 & 0 & I_{2} \\
0 & I_{1} & 0 & -I_{2} & 0 \\
0 & 0 & I_{1} & 0 & 0 \\
0 & -I_{2} & 0 & I_{3} & 0 \\
I_{2} & 0 & 0 & 0 & I_{3}
\end{array}\right]
$$

in which $I_{1}=\int \rho \mathrm{d} z ; I_{2}=\int \rho z \mathrm{~d} z ; I_{3}=\int \rho z * z \mathrm{~d} z$. Thus by substituting the expression for $\Pi$ and $E$ in eqn (46) we get the equation of motion as

$$
\begin{array}{r}
\delta\left(\int _ { t _ { 1 } } ^ { t _ { 2 } } \left[\frac{1}{2} \int_{A} \bar{\epsilon}_{x}^{t} D_{x} \bar{\epsilon}_{x} \mathrm{~d} A-\int_{A} \mathbf{d}_{x}^{t} \mathbf{F} \mathrm{d} A\right.\right. \\
\left.\left.-\frac{1}{2} \int_{A} \dot{\mathbf{d}}_{x}^{t} \mathbf{m} \dot{\mathbf{d}}_{x} \mathrm{~d} A\right]\right) \mathrm{d} t=0
\end{array}
$$

\section{FINITE ELEMENT DISCRETIZATION}

\subsection{Discretization in space}

In the standard finite element technique the solution domain is discretized into $N E$ subdomains (elements) such that

$$
\begin{aligned}
& \Pi(\mathbf{d})=\sum_{\ell=1}^{N E} \Pi^{e}(\mathbf{d}) \\
& E(\mathbf{d})=\sum_{i=1}^{N E} E^{e}(\mathbf{d}),
\end{aligned}
$$

where $\Pi$ and $\Pi^{e}$ are the total potential energies of the system and the element, respectively, and $E$ and $E^{u}$ are the kinetic energies of the system and element, respectively. The basic discretization is carried over an element only and the resulting equations are summed over the entire domain. 


\subsection{Displacement function}

The nodal and generalized displacement vectors are related with the help of shape functions as

$$
\begin{aligned}
& {\left[\begin{array}{c}
u_{o x} \\
v_{n x} \\
w_{o x} \\
\theta_{x x} \\
\theta_{y x}
\end{array}\right]=\sum_{i=1}^{N N}\left[\begin{array}{l}
\mathbf{N}_{i}(\xi, \eta) u_{i x} \\
\mathbf{N}_{i}(\xi, \eta) v_{i x} \\
\mathbf{N}_{i}(\xi, \eta) w_{i x} \\
\mathbf{N}_{i}(\xi, \eta) \theta_{i x x} \\
\mathbf{N}_{i}(\xi, \eta) \theta_{i x x}
\end{array}\right]} \\
& =\sum_{i=1}^{N N}\left[\begin{array}{ccccc}
\mathbf{N}_{i} & 0 & 0 & 0 & 0 \\
0 & \mathbf{N}_{i} & 0 & 0 & 0 \\
0 & 0 & \mathbf{N}_{i} & 0 & 0 \\
0 & 0 & 0 & \mathbf{N}_{i} & 0 \\
0 & 0 & 0 & 0 & \mathbf{N}_{i}
\end{array}\right] \quad\left[\begin{array}{c}
u_{i x} \\
v_{i x} \\
w_{i x} \\
\theta_{i x x} \\
\theta_{i x x}
\end{array}\right]
\end{aligned}
$$

i.e.

$$
\mathbf{d}_{x}=\sum_{l=1}^{N N} \mathbf{N}_{i} \mathbf{d}_{i x} .
$$

Let the displacement at node $i$ in the nodal coordinate system be

$$
\mathbf{d}_{i n}=\left(u_{m n}, v_{m}, w_{m n}, \theta_{\mathrm{k} n}, \theta_{y n}\right)^{t} .
$$

Using a transformation matrix $R_{z i}$ given in Appendix $\mathrm{D}$ we can write

$$
\mathbf{d}_{i z}=R_{x i} \mathbf{d}_{i n} .
$$

If we define

$$
\mathbf{d}_{\mathrm{n}}^{\mathrm{l}}=\left[\begin{array}{llllll}
u_{1 n} & v_{1 n} & w_{1 n} & \theta_{x i n} & \theta_{y ! n} & \cdots \\
u_{i n} & v_{i n} & w_{i n} & \theta_{x i n} & \theta_{y i n} & \cdots \\
u_{n n} & v_{n n} & w_{n n} & \theta_{x n n} & \theta_{y n n} &
\end{array}\right]
$$

and

$$
\mathbf{N}=\left[\begin{array}{ccccccccccccccccc}
N_{1} & 0 & 0 & 0 & 0 & - & \mathbf{N}_{i} & 0 & 0 & 0 & 0 & - & \mathbf{N}_{n !} & 0 & 0 & 0 & 0 \\
0 & N_{1} & 0 & 0 & 0 & - & 0 & \mathbf{N}_{i} & 0 & 0 & 0 & - & 0 & \mathbf{N}_{n n} & 0 & 0 & 0 \\
0 & 0 & N_{1} & 0 & 0 & - & 0 & 0 & \mathbf{N}_{i} & 0 & 0 & - & 0 & 0 & \mathbf{N}_{n n} & 0 & 0 \\
0 & 0 & 0 & N_{1} & 0 & - & 0 & 0 & 0 & \mathbf{N}_{i} & 0 & - & 0 & 0 & 0 & \mathbf{N}_{m} & 0 \\
0 & 0 & 0 & 0 & N_{1} & - & 0 & 0 & 0 & 0 & \mathbf{N}_{i} & - & 0 & 0 & 0 & 0 & \mathbf{N}_{m}
\end{array}\right]
$$

therefore

$$
\bar{\epsilon}_{m x}=\overline{\mathbf{B}}_{m}^{*} \mathbf{d}_{n} .
$$

Similarly

$$
\begin{aligned}
\overline{\boldsymbol{\epsilon}}_{m x} & =\mathbf{B}_{h}^{*} \mathbf{d}_{n} \\
\bar{\epsilon}_{m x} & =\overline{\mathbf{B}}_{s}^{*} \mathbf{d}_{n},
\end{aligned}
$$

where $\overline{\mathbf{B}}_{m}^{*}, \mathbf{B}_{b}^{*}, \overline{\mathbf{B}}_{s}^{*}$ are the strain matrices of the membrane, bending and shear displacements, respectively. The forms of $\mathbf{B}_{m x i}^{*}, \bar{B}_{b x i}^{*}, \mathbf{B}_{s x i}^{*}$ are given in Appendix E. Thus

$$
\bar{\epsilon}_{x}=\mathbf{B}^{*} \mathbf{d}_{n},
$$

where

$$
\mathbf{B}^{*}=\left[\overline{\mathbf{B}}_{m}^{*}, \overline{\mathbf{B}}_{b}^{*}, \overline{\mathbf{B}}_{s}^{*}\right]^{\prime}
$$

\subsection{Isoparametric representation}

In isoparametric representation the geometry and the displacement fields are interpolated using the

(63) same shape functions. The space coordinates are expressed as

$$
\left[\begin{array}{l}
x \\
y
\end{array}\right]=\sum_{i=1}^{N N}\left[\begin{array}{cc}
N_{i} & 0 \\
0 & N_{i}
\end{array}\right]\left[\begin{array}{l}
x_{i} \\
y_{i}
\end{array}\right]
$$

Then

$$
\mathbf{d}_{\mathrm{x}}=\sum_{i=1}^{N N} \mathbf{N}_{i} R_{x i} \mathbf{d}_{i n}=\mathbf{N} R \mathbf{d}_{n} .
$$

in which $x_{i}, y_{i}$ are coordinates of node $i$ and $N_{i} \mathrm{~s}$ are shape functions in terms of $\xi$ and $\eta$. In the present 
formulation we require the derivatives of the shape functions with respect to $x_{x}, y_{x}$ coordinates. The Jacobian matrix relates the area integral in $x_{x}, y_{x}$ coordinate to that in $\xi$ and $\eta$ coordinate system and is given by

$$
\mathbf{J}=\left|\begin{array}{cc}
\frac{\partial x_{x}}{\partial \xi} & \frac{\partial y_{x}}{\partial \xi} \\
\partial x_{x} & \partial y_{x} \\
\partial \eta & \partial \eta
\end{array}\right|
$$

After adopting the chain rule we get the Jacobian matrix as similarly

$$
\begin{aligned}
& \mathbf{K}_{h}^{e}=\sum_{\beta=1}^{m} w_{\beta}\left(\overline{\mathbf{B}}_{h}^{* t}\right)_{\beta}\left(\overline{\mathbf{D}}_{h x}\right)_{\beta}\left(\overline{\mathbf{B}}_{h}^{*}\right)_{\beta}|\mathbf{J}|_{\beta} \\
& \mathbf{K}_{s}^{e}=\sum_{\beta=1}^{m} w_{\beta}\left(\overline{\mathbf{B}}_{s}^{* \prime}\right)_{\beta}\left(\overline{\mathbf{D}}_{s z}\right)_{\beta}\left(\overline{\mathbf{B}}_{s}^{*}\right)_{\beta}|\mathbf{J}|_{\beta} .
\end{aligned}
$$

Here $\mathbf{K}_{m}^{e}, \mathbf{K}_{b}^{e}, \mathbf{K}_{s}^{e}$ are the element membrane, bending and shear stiffness matrices, respectively, $m$ is the number of Gauss points and $w_{\beta}$ are the weights of Gauss points. Thus, any order of integration (either

$$
\mathbf{J}=\left|\begin{array}{ll}
l_{1 x} \frac{\partial X}{\partial \xi}+m_{1 x} \frac{\partial Y}{\partial \xi}+n_{1 x} \frac{\partial Z}{\partial \xi}, & l_{2 x} \frac{\partial X}{\partial \xi}+m_{2 x} \frac{\partial Y}{\partial \xi}+n_{2 x} \frac{\partial Z}{\partial \xi} \\
l_{1 x} \frac{\partial X}{\partial \eta}+m_{1 x} \frac{\partial Y}{\partial \eta}+n_{1 x} \frac{\partial Z}{\partial \eta}, & l_{2 x} \frac{\partial X}{\partial \eta}+m_{2 x} \frac{\partial Y}{\partial \eta}+n_{2 x} \frac{\partial Z}{\partial \eta}
\end{array}\right|
$$

where $l_{1 x}, m_{1 x}, n_{1 x}$ and $l_{2 x}, m_{2 x}, n_{2 x}$ are the direction cosines of the local $\mathbf{i}_{x}$ and $\mathbf{j}_{x}$ axes with respect to global $\mathbf{i}, \mathbf{j}, \mathbf{k}$, respectively. An elemental area on the midsurface is given as

$$
\int_{A} \mathrm{~d} A=\int_{A} \mathrm{~d} x_{x} \mathrm{~d} y_{x}=\int_{-1}^{1} \int_{-1}^{1}|\mathbf{J}| \mathrm{d} \xi \mathrm{d} \eta .
$$

\subsection{Element stiffness matrix}

From eqn (53) we get the expression of the strain energy for an element

$$
U^{e}=\frac{1}{2} \int_{A} \bar{\epsilon}_{x}^{c r} \overline{\mathbf{D}}_{x}^{e} \bar{\epsilon}_{x}^{e} \mathrm{~d} A
$$

Using eqn (73) and substituting into the above equation

$$
\begin{aligned}
U^{e} & =\frac{1}{2} \int_{A} \mathbf{d}_{n}^{e} \mathbf{B}^{*} \overline{\mathbf{D}}_{x}^{e} \mathbf{B}^{*} \mathbf{d}_{n}^{e} \mathrm{~d} A \\
& =\frac{1}{2} \mathbf{d}_{n}^{e} \mathbf{K}^{e} \mathbf{d}_{n}^{e} .
\end{aligned}
$$

Here $\mathbf{K}^{*}$ is the element stiffness matrix and is given by

$$
\mathbf{K}^{e}-\int_{A} \mathbf{B}^{* \prime} \overline{\mathbf{D}}_{\mathrm{x}}^{e} \mathbf{B}^{*} \mathrm{~d} A
$$

using eqn (74) and (42) and simplifying the expression

$$
\mathbf{K}^{e}=\mathbf{K}_{m}^{e}+\mathbf{K}_{h}^{p}+\mathbf{K}_{s}^{e},
$$

where

$$
\begin{aligned}
& \mathbf{K}_{m}^{e}=\int_{-1}^{+1} \int_{-1}^{+1} \overline{\mathbf{B}}_{m}^{* \prime} \overline{\mathbf{D}}_{m z} \mathbf{B}_{m}^{*}|\mathbf{J}| \mathrm{d} \xi \mathrm{d} \eta \\
& \mathbf{K}_{m}^{\mathrm{e}}=\sum_{\beta=1}^{m} w_{\beta}\left(\mathbf{B}_{m}^{*}\right)_{l \beta}\left(\mathbf{\mathbf { D }}_{m \times}\right)_{\beta}\left(\mathbf{\mathbf { B }}_{m}^{*}\right)_{\beta}|\mathbf{J}|_{\beta},
\end{aligned}
$$

$2 \times 2$ or $3 \times 3$ ) can be independently applied to calculate any of the individual $K$ matrix.

\subsection{Element mass matrix}

This is derived from the kinetic energy expression. Using eqn (51)

$$
E^{c}=\frac{1}{2} \int_{A} \mathbf{d}_{x}^{e e} \mathbf{m d} \mathbf{d}_{2}^{c} \mathrm{~d} A,
$$

from eqn (65)

$$
\mathbf{d}_{z}^{e}=\mathbf{N} R \mathbf{d}_{n}^{e},
$$

substituting in eqn (88)

$$
\begin{aligned}
E & =\frac{1}{2} \int_{A} \mathbf{d}_{n}^{\mathbf{d}^{\prime}} R^{\prime} \mathbf{N}^{\prime} \mathbf{m N} R \mathbf{d}_{n}^{c} \mathrm{~d} A \\
& =\frac{1}{2} \mathbf{d}_{n}^{c} \mathbf{M} \mathbf{M}^{\prime} \mathbf{d}_{n}^{c},
\end{aligned}
$$

where $\mathbf{M}^{r}$ is the element mass matrix and is given by

$$
\mathbf{M}^{e}=\int_{A} R^{\prime} \mathbf{N}^{\prime} \mathbf{m} \mathbf{N} R \mathrm{~d} A,
$$

$\mathbf{m}$ and $\mathbf{N}$ are inertia and shape function matrices, respectively. The consistent mass matrix $\mathbf{M}^{r}$ is evaluated using the $3 \times 3$ Gauss quadrature rule as follows:

$$
\begin{aligned}
\mathbf{M}_{i j}^{c} & =\int_{-1}^{+1} \int_{-1}^{+1} R_{i}^{\prime} \mathbf{N}_{i}^{\prime} \mathbf{m} \mathbf{N}_{i} R_{i}|\mathbf{J}| \mathrm{d} \xi \mathrm{d} \eta \\
& =\sum_{u=1}^{p} \sum_{k=1}^{p} \mathfrak{w}_{i}^{\prime} \mathfrak{w}_{b}^{\prime}|\mathbf{J}| R_{i}^{\prime} \mathbf{N}_{i}^{\prime} \mathbf{m} \mathbf{N}_{i} R_{i}, \quad i, j=1, N N,
\end{aligned}
$$

where $p$ is the number of Gauss points in any one direction and $w_{a}, w_{h}$ are corresponding weights. A 
special mass lumping procedure which is given in [12] or is used here.

\subsection{Element load vector}

This can be derived from the expression of work done by external forces. From eqn (53), we get

$$
W^{e}=\int \mathbf{d}_{x}^{e t} \mathbf{F} \mathrm{d} A
$$

Using eqn (65) we get

$$
W^{e}=\int_{A} \mathbf{d}_{x}^{e t} \mathbf{F} \mathrm{d} A=\mathrm{d}_{n}^{e t} \mathbf{f}_{\varepsilon},
$$

where

$$
\mathbf{f}^{\prime}=\int_{A} R^{\prime} \mathbf{N}^{\prime} \mathbf{F} \mathrm{d} A,
$$

F is a matrix containing magnitude of forces in each of five degrees of freedom direction.

\subsection{Discrete equations of motion}

Using eqn (47), we can write

$$
\Pi^{e}=U^{e}-W^{e}
$$

Here $\Pi^{r}$ is the total potential energy of an element $e$ and $U^{e}, W^{e}$ are the internal strain energy and external work done, respectively. From eqn (81) we get

$$
U^{e}=\frac{1}{2} \mathbf{d}_{n}^{\mathbf{e}^{\prime}} \mathbf{K}^{\boldsymbol{e}} \mathbf{d}_{n}^{e}
$$

The first variation of the internal strain energy can be written in matrix form as

$$
\delta U_{e}=\delta \mathbf{d}_{n}^{\ell \ell} \mathbf{K}^{e} \mathbf{d}_{n}^{e}
$$

Here $\mathbf{K}^{e}$ is the element stiffness matrix. The first variation of the external work done on the element can be written in matrix form using eqn (96) as

$$
\delta W^{e}=\delta \mathbf{d}_{n}^{e} \mathbf{f}^{e}
$$

$f^{\prime}$ is the element load vector. Also the first variation of kinetic energy $\delta E^{\prime}$ for an element can be written in matrix form using eqn (91) as

$$
\delta E^{e}=-\delta \mathbf{d}_{n}^{e t} \mathbf{M}^{*} \ddot{\mathbf{d}_{n}^{\prime}}
$$

Here $\mathbf{d}_{n}^{e}$ is vector of element nodal accelerations. Substituting the above expressions in eqn (46) we get the following form of Hamilton's equation

$$
\int_{l_{1}}^{t_{2}}\left(\delta U^{v}-\delta W^{e}-\delta E^{e}\right) \mathrm{d} t=0
$$

$$
\int_{t_{1}}^{t_{2}} \delta \mathbf{d}_{n}^{e t}\left[\mathbf{M}^{e} \mathbf{d}_{n}^{e}+K^{\epsilon} \mathbf{d}_{n}^{e}-\mathbf{f}_{n}^{e}\right] \mathrm{d} t=0 .
$$

Since this relation is valid for every virtual displacement $\delta \mathbf{d}_{n}^{e}$ we get

$$
\mathbf{M}^{e} \ddot{\mathbf{d}}_{n}^{e}+\mathbf{K}^{e} \mathbf{d}_{n}^{e}=\mathbf{f}_{n}^{e}
$$

This is a finite element equation of motion for one element of domain. These element equations are now assembled as in equations (54) and (55) to yield the global equation of motion for the entire domain, i.e.

$$
\mathbf{M} \mathbf{d}+\mathbf{K d}=\mathbf{f}
$$

Here $\mathbf{d}, \overrightarrow{\mathbf{d}}$ are the global vectors of unknown displacement and acceleration, respectively. $\mathbf{M}, \mathbf{K}, \mathbf{f}$ are global mass, stiffness and nodal load vectors, respectively, and are given as

$$
\begin{aligned}
\mathbf{K} & =\sum_{e=1}^{N E} \mathbf{K}^{e} \\
\mathbf{M} & =\sum_{e=1}^{N E} \mathbf{M}^{e} \\
\mathbf{f} & =\sum_{e=1}^{N E} \mathbf{f}^{e}
\end{aligned}
$$

\subsection{Seismic input}

In the case of seismic input problems the load vector is of the following form in the equation of motion (106)

$$
\mathbf{f}=-\mathbf{M r} \ddot{i}_{g}
$$

Here $\mathbf{M}$ is the mass matrix of the system as derived earlier, $\mathbf{r}$ is the influence matrix which is a column matrix containing ones and zeros, $\ddot{\mathbf{u}}_{g}$ is the ground acceleration input which varies with time.

\section{NUMERICAL RESULTS AND DISCUSSION}

\subsection{Preliminary remarks}

Nine-noded biquadratic Lagrangian elements with five degrees of freedom per node are used for the analysis. Numerical quadrature is used for the evaluation of the element matrices. A $2 \times 2$ Gauss quadrature rule is employed to evaluate the shear energy term, while a $3 \times 3$ Gauss rule is utilized for all the remaining terms in stiffness, mass and load matrices. Zero initial conditions are assumed in all the cases. All the computations are carried out in single precision on a CDC Cyber 180/840 system with 16 significant digits accuracy. Nodal boundary conditions are prescribed. The results from the software SHELDYNA (program for mode superposition analysis), and TIME (program for time integration 
analysis) developed by the authors are compared with BLAST (program for elasto-dynamic analysis of shells using assumed strain degenerate shell element developed by Huang [13]).

\subsection{Boundary conditions and problem data}

The following boundary conditions are employed

1. Fixed $u_{n}=0, v_{n}=0, w_{n}=0, \theta_{v n}=0, \theta_{y n}=0$.

2. SSI (simply supported diaphragm): $y=$ const, $u_{n}=0, w_{n}=0, \theta_{v n}=0 ; x=$ const, $v_{n}=0, w_{n}=0$. $\theta_{x z}=0$.

3. SS2 (simply supported hinged): $y=$ const, $u_{n}=0, v_{n}=0, w_{n}=0, \theta_{1 n}=0, x=$ const. $u_{n}=0$, $v_{n}=0, w_{n}=0, \theta_{x m}=0$.

4. Symmetric: $y=$ const, $v_{n}=0, v_{v}=0: x=$ const, $u_{n}=0, \theta_{m}=0$.

The following sets of data are used for the numerical examples analysed in this section.

Data 1: A deep thin arch-this example is taken from [14]

radius $(R)=67.115 \mathrm{in}$, thickness $(h)=1.0 \mathrm{in}$ central angle $(\beta)=15$, width $(b)=1.0$ in density $(\rho)=2.44 \times 10^{-4} \mathrm{lb} \mathrm{sec}^{2} / \mathrm{in}^{4}$

Young's modulus $(E)=1.0 \times 10^{7} \mathrm{lb} / \mathrm{in}^{2}$

Poisson's ratio $(v)=0.3$,

load intensity $(p)=0.25 \mathrm{bb} / \mathrm{in}^{2}$.

Data 2: A deep thin spherical cap this example is taken from [13]

radius $(R)=20.0 \mathrm{in}$, thickness $(h)=0.3 \mathrm{in}$ central angle $(\beta)=120$.

density $(\rho)=7.33 \times 10^{-4} \mathrm{lb} \mathrm{sec}^{2} / \mathrm{in}^{4}$,

Poisson's ratio $(v)=0.3$

Young's modulus $(E)=3.0 \times 10^{7} \mathrm{lb} / \mathrm{in}^{2}$

load intensity $(p)=600 \mathrm{lb} / \mathrm{in}^{2}$.

Data 3: A thin cylindrical diaphragm-this example is taken from [15]

radius $(R)=300.0 \mathrm{in}$, thickness $(h)=0.3 \mathrm{in}$ central angle $(x)=40$, length $(L)=100.0 \mathrm{in}$ density $(p)=2.25 \times 10^{7} \mathrm{k} \mathrm{sec}^{2} / \mathrm{in}^{4}$,

Young's modulus $(E)=3.6 \times 10^{3} \mathrm{kip} / \mathrm{in}^{2}$

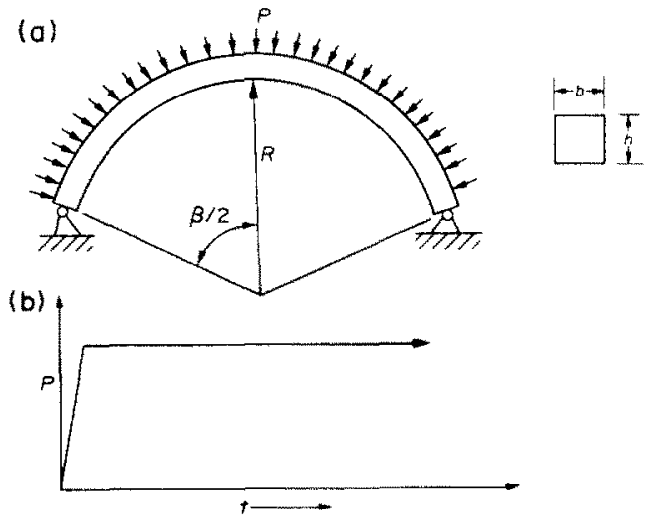

Fig. 2. A deep thin arch subjected to impulsive u.d.l.

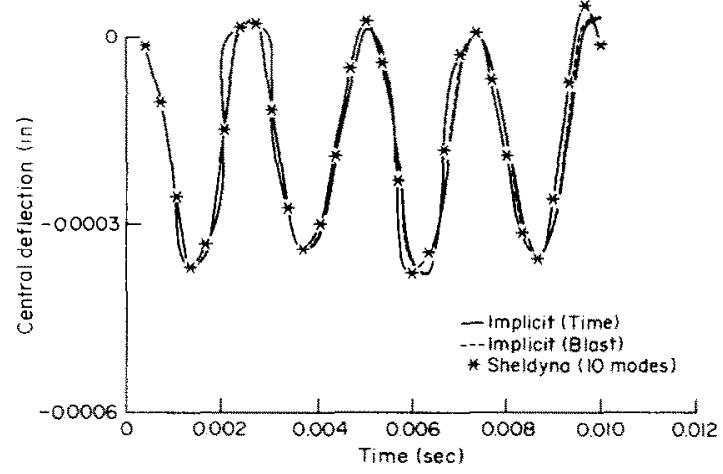

Fig. 3. A thin arch subjected to suddenly applied u.d.I.

Poisson's ratio $(v)=0.15$

vertical acceleration $\left(u_{Q}\right)=115.9 \mathrm{in} / \mathrm{sec}^{2}$.

Data 4: Briones dam intake tower-this example is taken from [16]

height $(L)=230 \mathrm{ft}$

inner diameter at bottom (elevation 360.0 ) $=20 \mathrm{ft}$

inner diameter at top (elevation 575.0) $=10 \mathrm{ft}$

thickness at bottom $=1.33 \mathrm{ft}$

thickness at elevation $575,0=1.113 \mathrm{ft}$

density $(\rho)=4.18 \mathrm{lb} \mathrm{sec}^{2} / \mathrm{in}^{4}$

Young's modulus $(E)=6.48 \times 10^{8} \mathrm{lb} / \mathrm{in}^{2}$

Poisson's ratio $(v)=0.17$

\subsection{Examples and discussion}

Example 1. A deep thin circular arch of radius 67.115 in and unit cross-sectional area with a eentral angle of 15 is modelled in this case using shell elements. It is simply supported (SS2) at the ends and subjected to an impulsive step loading in the form of a uniformly applied pressure over its span as shown in Fig. 2. This has been analysed by SHELDYNA using the first ten modes. The same has also been analysed by TIME and BLAST using an implicit time scheme with a time step of $3.315 \times 10^{5} \mathrm{sec}$. The results have been recorded in the form of transient variation of central deficction as shown in Fig. 3.

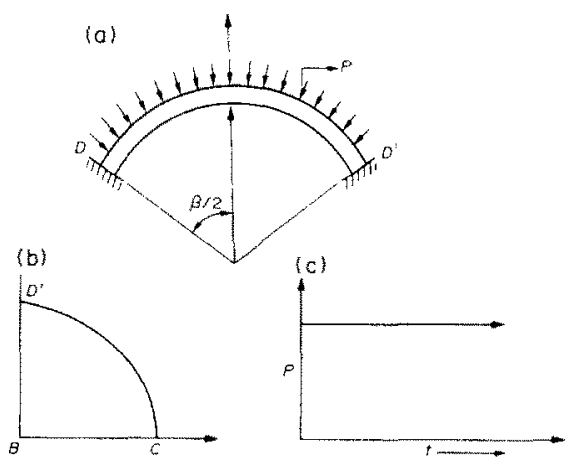

Fig. 4. A deep thin spherical cap subjected to suddenly applied pressure. 


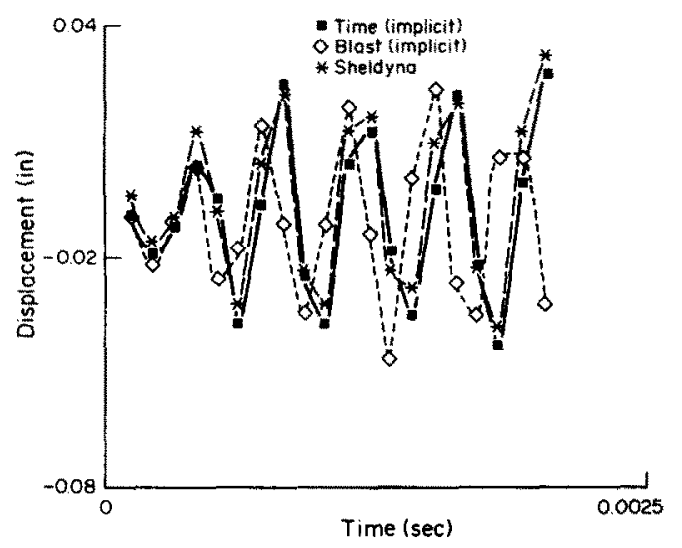

Fig. 5. Variation of central deflection of a thin shell cap subjected to suddenly applied pressure.

Uniform periodic variation of the deflection is observed. The maximum value of the central deflection observed is $3.9 \times 10^{-5}$ in all the three cases. It can be seen from Fig. 3 that the results by all the methods are in good agreement.

Example 2. In this example a deep thin spherical cap, shown in Figs 4(a) and (b), is analysed. This is clamped all around its boundary. This is subjected to a suddenly applied uniform pressure of intensity $600 \mathrm{lb} / \mathrm{in}^{2}$, the variation of which is shown in Fig. 4(c). Taking advantage of symmetry, only one quarter of the shell is taken and is discretized using 12 shell elements. In the analysis by SHELDYNA the first ten modes were considered. A time step of $2 \times 10^{-5} \mathrm{sec}$ was adopted in the analysis using TIME and BLAST. The transient variation of the central deflection has been recorded in the form of a graph as shown in Fig. 5. The maximum central deflection observed is $3.5 \times 10^{-2} \mathrm{in}$. The closeness of the results by TIME and BLAST is clearly depicted in Fig. 5. A
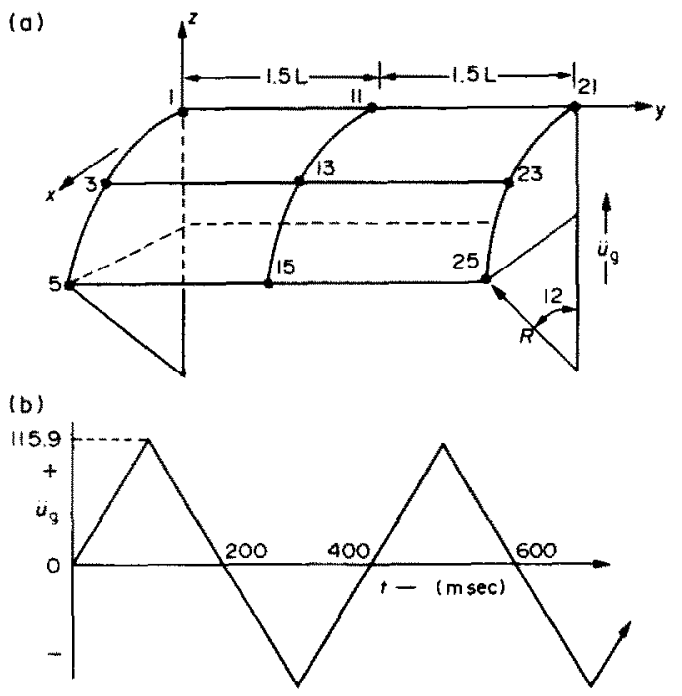

Fig. 6. A thin cylindrical cap subjected to base acceleration.

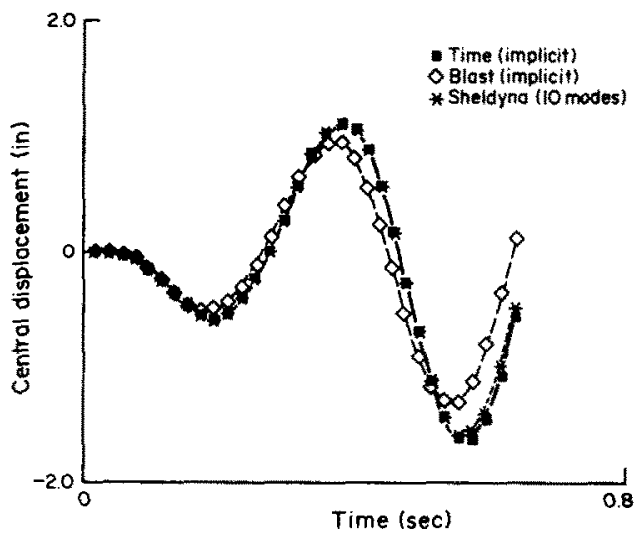

Fig. 7. Variation of deflection of an edge point of a thin cylindrical cap subjected to vertical acceleration.

slight difference in the results by SHELDYNA is observed. It is expected that improved convergence will be achieved by increasing the number of modes.

Example 3. In this case a thin cylindrical cap as shown in Fig. 6(a) is analysed for the vertical base acceleration shown in Fig. 6(b). This is simply supported (SS1) along the curved edge. Taking symmetry into account again only one quarter of the structure is discretized using four shell elements. The loading is

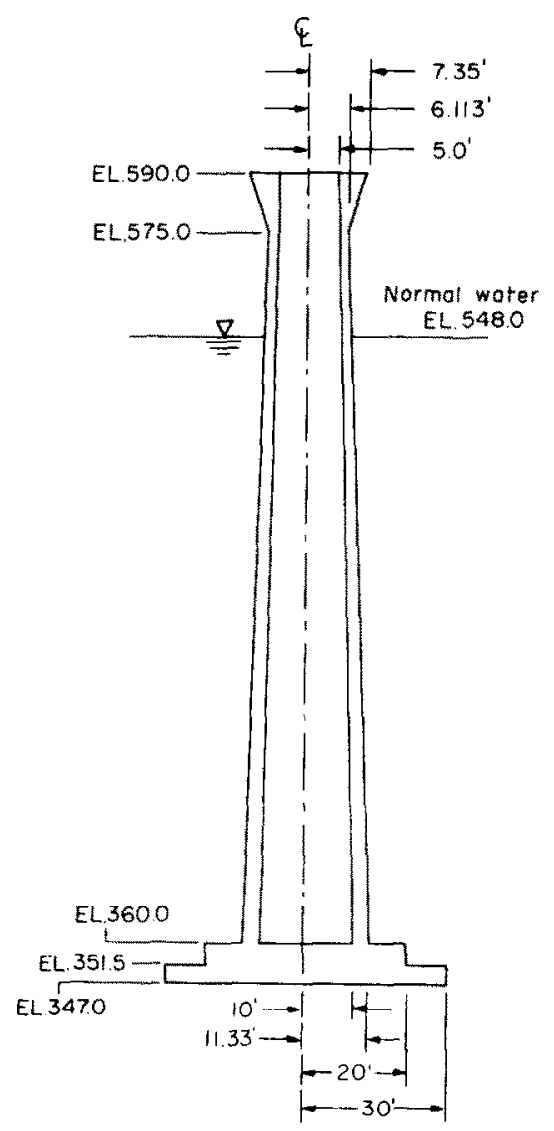

Fig. 8. Brione"s dam intake tower. 


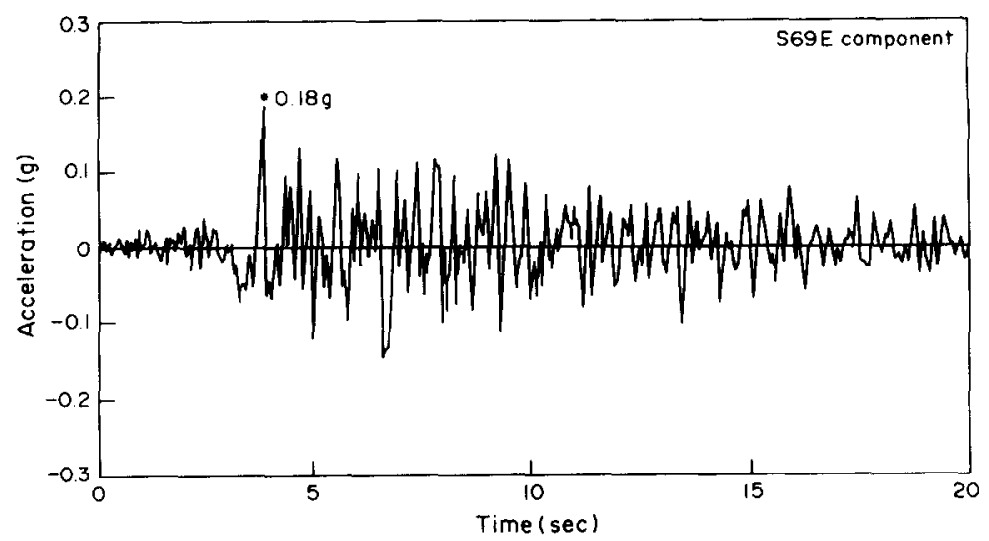

Fig. 9. Ground motion recorded at Taft Lincoln School Tunnel, California, earthquake, 21 July 1952.

in the form of vertical ground acceleration which varies as a saw-tooth wave as shown in Fig. 6(b). The variation of the vertical deflection of the corner edge point (node 5) is shown in Fig. 7. In this case also the first ten modes were considered while analysing by SHELDYNA and in the case of analysis by TIME and BLAST a time-step of value $0.005 \mathrm{sec}$ was used. The maximum value of the deflection observed is 1.63 in in the case of SHELDYNA and TIME and 1.53 in in the case of BLAST. It is observed in Fig. 7 that the variation is more or less similar in all the cases.

Example 4. In this example a Briones dam intake tower, as shown in Fig. 8 and adopted from [16], has been analysed. The tower tapers with an internal radii of $10 \mathrm{ft}$ at bottom to $5 \mathrm{ft}$ at the top. The effective height of the tower is $230 \mathrm{ft}$. This has been analysed as a hollow tapering cylindrical cantilever fixed at the base. This is subjected to an earthquake input of S69E component recorded by the Taft Lincoln School Tunnel, California, as shown in Fig. 9. Due to unidirectional symmetry only one half of the structure is considered and is discretized using 11 shell elements along the axis. The analysis is done for the 'no water condition' only. In the analysis using

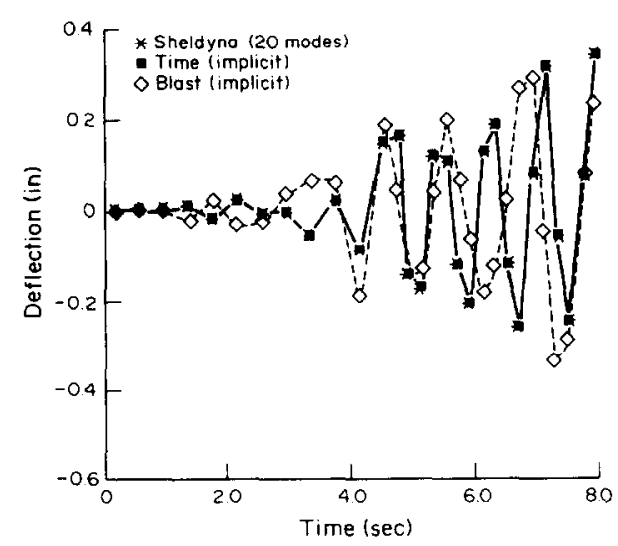

Fig. 10. Variation of tip deflection of a chimney subjected to seismic input of Fig. 9.
SHELDYNA the first 20 modes were considered and a time-step of $0.01 \mathrm{sec}$ was taken in the case of analysis by TIME and BLAST. The variation of the tip deflection is recorded as an output in Fig. 10. In the actual case this was analysed by taking it as a cantilever beam and was discretized using beam elements. Five percent damping was considered in each mode and first five modes were taken. The maximum displacement observed was $3.0 \mathrm{in}$. The maximum deflection observed in the present case with no damping being considered is $4.5 \mathrm{in}$. It can be seen from the results that although the results differ in the initial stage slightly, they are more or less similar in the later stage by all the three approaches.

\section{CONCLUSIONS}

The Mindlin-Reissner theory is employed with explicitly integrated three-dimensional degenerated shell elements for undamped elasto-dynamic as well as seismic analysis of shells. Excellent agreement between the results by SHELDYNA (software developed by authors for mode superposition) and TIME (software developed by authors for time integration) is observed both in the case of dynamic loading as well as in base input problems. Also the closeness of results with BLAST (software developed by Huang for clasto-dynamic analysis using assumed strain degenerate shell element) shows the accuracy and efficiency of the present explicitly integrated element. For the same level of accuracy, a considerable saving in computation time is achieved in the mode superposition as compared to time integration methods since only the first few modes were sufficient to obtain the results with the same accuracy. As the formulation is on a nodal hasis the imposition of boundary conditions is found to be straightforward.

\section{REFERENCES}

1. S. Ahmad, B. M. Irons and O. C. Zienkiewicz, Analysis of thick and thin shell structures by curved finite elements. Int. J. Numer. Meth. Engng 2, 419451 (1970). 
2. O. C. Zienkiewicz, R. L. Taylor and J. M. Too, Reduced integration technique in general analysis of plates and shells. Int. J. Numer. Meth. Engng 3, 275-290 (1971).

3. D. S. Malkus and T. J. R. Hughes, Mixed finite element methods-reduced and selective techniques: a unification of concepts. Comput. Meth. appl. Mech. Engng 15, 63-81 (1978).

4. T. J. R. Hughes, M. Cohen and M. Haroun, Reduced and selective integration techniques in finite element analysis of plates. Nucl. Engng Des. 46, 203-222 (1978).

5. E. D. Pugh, E. Hinton and O. C. Zienkiewicz, A study of quadrilaterial plate bending elements with reduced integration. Int. J. Numer. Meth. Engng 12, 1059-1079 (1978).

6. H. Parisch, A critical survey of the 9-node degenerate shell element with special emphasis on thin shell application and reduced integration. Comput. Meth. appl. Mech. Engng 20, 323-350 (1979).

7. T. Belytschko, W. K. Liu and J. S. J. Ong, Implementation and application of a 9-node Lagrangian shell element with spurious mode control. Comput. Struct. 20, 121-128 (1985).

8. R. V. Milford and W. C. Schnobrich, Degenerated isoparametric finite element using explicit integration. Int. J. Numer. Meth. Engng 23, 133-154 (1986).
9. T. Kant and D. Datye, Finite elements available for the analysis of curved thin-walled structures. In Finite Element Applications to Thin-walled Structures (Edited by J. W. Bull). Elsevier, London (1990).

10. K. J. Bathe and E. N. Dvorkin, A four node plate bending element based on Mindlin/Reissner plate theory and a mixed interpolation. Int. J. Numer. Meth. Engng 21, 367-383 (1985).

11. H. C. Huang and E. Hinton, A new nine-node degenerate shell element with enhanced membrane and shear interpolation. Int. J. Numer. Meth. Engng 22, 73-92 (1986).

12. E. Hinton, T. Rock and O. C. Zienkiewicz, A note on mass lumping and related processes in the finite element method. J. Earth. Engng Struct. Dyn. 4, 245- 249 (1976).

13. H. C. Huang, Static and Dynamic Analysis of Shells. Springer, London (1989).

14. K. J. Bathe, Static and dynamic geometric and material non-linear analysis. Ph.D. thesis, University of California at Berkeley (1974).

15. W. Weaver and P. R. Johnston, Structural Dynamics by Finite Elements. Prentice-Hall, Englewood Cliffs, NJ (1987).

16. A. K. Goel, Earthquake analysis and response of inlet outlet towers. Ph.D. thesis, University of California at Berkeley (1988).

\section{APPENDIX A}

The constitutive matrix $\mathbf{C}$ is given below

$$
\mathbf{C}=\left[\begin{array}{ccccc}
\frac{E}{(1-v v)} & \frac{v E}{(1-v v)} & 0 & 0 & 0 \\
\frac{v E}{(1-v v)} & \frac{E}{(1-v v)} & 0 & 0 & 0 \\
0 & 0 & \frac{E}{2(1+v)} & 0 & 0 \\
0 & 0 & 0 & \frac{E}{2(1+v)} & 0 \\
0 & 0 & 0 & 0 & \frac{E}{2(1+v)}
\end{array}\right] .
$$

APPENDIX B

The $\mathbf{D}$ matrices in the membrane, bending and shear elements are given below

$$
\begin{aligned}
& \mathbf{D}_{m x}=\left[\begin{array}{cccccccc}
\frac{E t_{x}}{(1-v v)} & \frac{v E t_{x}}{(1-v v)} & 0 & 0 & 0 & 0 & 0 & 0 \\
\frac{v E t_{x}}{(1-v v)} & \frac{E t_{x}}{(1-v v)} & 0 & 0 & 0 & 0 & 0 & 0 \\
0 & 0 & \frac{E t_{x}}{2(1+v)} & 0 & 0 & 0 & 0 & 0
\end{array}\right] \\
& \mathbf{D}_{l, x}=\left[\begin{array}{cccccccc}
0 & 0 & 0 & \frac{E t_{x}^{3}}{12(1-v v)} & \frac{v E t_{x}^{3}}{12(1-v v)} & 0 & 0 & 0 \\
0 & 0 & 0 & \frac{v E t_{x}^{3}}{12(1-v v)} & \frac{E t_{x}^{3}}{12(1-v v)} & 0 & 0 & 0 \\
0 & 0 & 0 & 0 & 0 & \frac{E t_{x}^{3}}{24(1+v)} & 0 & 0
\end{array}\right] \\
& \mathbf{D}_{v \gamma}=\left[\begin{array}{cccccccc}
0 & 0 & 0 & 0 & 0 & 0 & \frac{E t_{x}}{2.4(1+v)} & 0 \\
0 & 0 & 0 & 0 & 0 & 0 & 0 & E l_{x} \\
2.4(1+v)
\end{array}\right]
\end{aligned}
$$




\section{APPENDIX C}

The $\mathbf{L}$ matrices for the membrane, bending and shear elements are given below

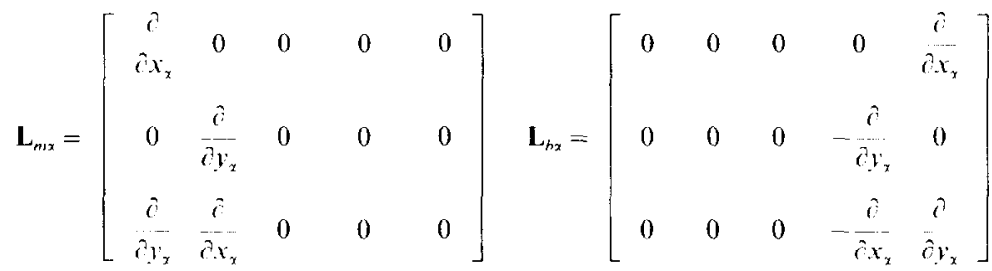

$$
\begin{aligned}
& \mathbf{L}_{s x}=\left[\begin{array}{ccccc}
0 & 0 & \frac{\partial}{\partial x_{x}} & 0 & 1 \\
0 & 0 & \frac{\partial}{\partial y_{x}} & -1 & 0
\end{array}\right]
\end{aligned}
$$

APPENDIX D

The local to nodal transformation matrix at a node $i$ is

$$
R_{x i}=\left[\begin{array}{ccccc}
\mathbf{i}_{x} \cdot \mathbf{i}_{n} & \mathbf{i}_{x} \cdot \mathbf{j}_{n} & \mathbf{i}_{x} \cdot \mathbf{k}_{n} & 0 & 0 \\
\mathbf{j}_{x} \cdot \mathbf{i}_{n} & \mathbf{j}_{x} \cdot \mathbf{j}_{n} & \mathbf{j}_{x} \cdot \mathbf{k}_{n} & 0 & 0 \\
\mathbf{k}_{x} \cdot \mathbf{i}_{n} & \mathbf{k}_{x} \cdot \mathbf{j}_{n} & \mathbf{k}_{x} \cdot \mathbf{k}_{n} & 0 & 0 \\
0 & 0 & 0 & \mathbf{i}_{x} \cdot \mathbf{i}_{n} & \mathbf{i}_{x} \cdot \mathbf{j}_{n} \\
0 & 0 & 0 & \mathbf{j}_{x} \cdot \mathbf{i}_{n} & \mathbf{j}_{x} \cdot \mathbf{j}_{n}
\end{array}\right] .
$$

\section{APPENDIX E}

The $\mathbf{B}$ matrices for membrane, bending and shear are

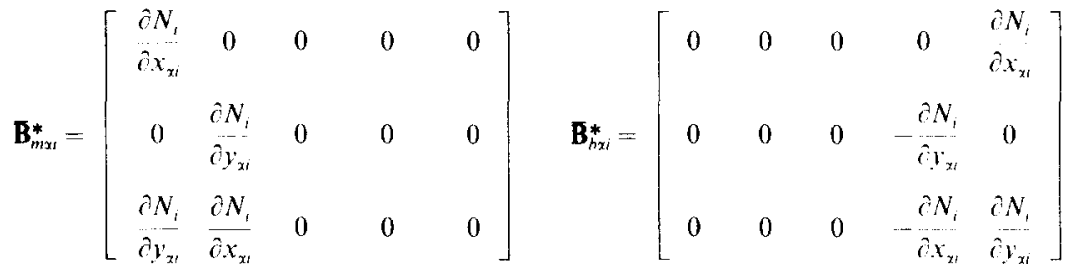

$$
\begin{aligned}
& \mathbf{B}_{s x i}^{*}=\left[\begin{array}{ccccc}
0 & 0 & \partial N_{i} & 0 & N_{i} \\
& & \partial x_{x i} & & \\
0 & 0 & \partial N_{i} & -N_{i} & 0
\end{array}\right]
\end{aligned}
$$

